\title{
Impurity effects on the quasiparticle spectrum of the Fulde-Ferrell-Larkin-Ovchinnikov state of a $d$-wave superconductor
}

\author{
Qian Wang, ${ }^{1}$ Chia-Ren Hu, ${ }^{2}$ and Chin-Sen Ting ${ }^{1}$ \\ ${ }^{1}$ Texas Center for Superconductivity, University of Houston, Houston, Texas 77204, USA \\ ${ }^{2}$ Department of Physics, Texas A\&M University, College Station, Texas 77843, USA
}

(Received 16 May 2006; published 1 December 2006)

\begin{abstract}
In a $d$-wave superconductor ( $d$-SC), a unitary impurity can induce a near-zero-energy resonant peak in the local tunneling density of states (LTDOS) due to the sign change of the order parameter (OP) on the Fermi surface. If a $d$-SC is quasi-two-dimensional, a large parallel magnetic field can drive it into the Fulde-FerrellLarkin-Ovchinnikov state, with an OP also changing sign in the real space like a checkerboard pattern. This double sign change leads to very subtle effects caused by a unitary impurity in the LTDOS for two locally stable locations of the impurity.
\end{abstract}

DOI: 10.1103/PhysRevB.74.212501

PACS number(s): 74.81.- g, 74.25.Ha, 74.50.+r

In a homogeneous, non- $s$-wave, singlet superconductor (SC), a unitary, nonmagnetic impurity can induce several quasilocalized, near-zero-energy (relative to the Fermi energy) resonant states (NZERSs), which are the direct consequence of the sign change of the superconducting order parameter (OP) on the Fermi surface. ${ }^{1-6}$ These states are responsible for the pair-breaking effects of nonmagnetic impurities in unconventional (i.e., non-s-wave) SCs, and they can lead to a near-zero-bias resonant peak (NZBRP) in the local tunneling density of states (LTDOS) near the impurity. This peak appears near the minimum of the bulk DOS of such a SC, and is one of the clearest pieces of evidence for unconventional pairing in, for example, high- $T_{c}$ SCs. These resonant states are close kin of the so-called zero-energy Andreev bound states (ZEABSs, also known as the midgap states $^{7}$ ) which form at properly oriented surfaces and interfaces of non- $s$-wave SCs, and are responsible for the zerobias conductance peaks observed ubiquitously in various types of tunneling experiments performed on various kinds of unconventional SCs. These ZEABSs are also the direct consequence of the sign change of the OP on the Fermi surface, and have in fact a topological origin. ${ }^{8}$ On the other hand, if a SC is quasi-two-dimensional, a strong magnetic field applied parallel to its layers can cause a large Zeeman splitting between its spin-up and spin-down electrons, and the orbital effect of the magnetic field can be suppressed. Then an inhomogeneous SC state known as the FuldeFerrell-Larkin-Ovchinnikov (FFLO) state ${ }^{9}$ can become energetically more favorable. We have recently shown ${ }^{10}$ that in an $s$-wave SC the OP of the FFLO state changes sign periodically in real space along one direction, with a periodic array of parallel real-space nodal lines, whereas in a

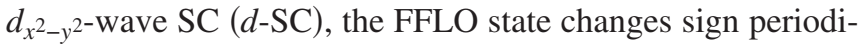
cally in two mutually perpendicular directions, forming a checkerboard pattern, with two mutually perpendicular sets of parallel real-space nodal lines, which are along the nodal directions the $d$-wave OP in the (relative) momentum space, i.e., at $45^{\circ}$ with the $a$ and $b$ axes. Right along these realspace nodal lines, but away from the saddle points where real-space nodal lines cross, ZEABSs can also form for the same topological reason. ${ }^{10}$ Then, as isolated impurities are added into a $d$-SC in such a state, two types of near- zero-energy quasiparticle states can potentially form-the ZEABSs localized near the real-space nodal lines, and the NZERSs localized near the impurities. ${ }^{11}$ The possible mutual interaction of these two types of states then constitutes an extremely interesting and fundamental topic, if only these two types of states are not far apart, so that their wave functions can overlap. Questions that can be raised include the following. Do they produce overlapping peaks in the LTDOS? Or there is some sort of level repulsion, but then how? (Would both types move to finite energies, or just one type?) Can one type preclude the existence of the other type? We shall see that the answer depends on the location of the impurity, and the results are quite unexpected. Confirming these results experimentally should then constitute some of the strongest evidence for the FFLO state in a $d$-SC. The FFLO state may very likely have been realized in the heavyfermion compound $\mathrm{CeCoIn}_{5},{ }^{12}$ which is very likely a $d$-SC, ${ }^{13}$ although no evidence presented so far for the FFLO state is direct.

To perform such a theoretical study, we need to first determine the locally stable locations of the impurity. (At very low concentrations of impurities, the spatial structure of the OP will destort slightly so that all impurities will be at such locations. It will not be true at higher concentrations, which will be studied in a future work.) We again solve the discrete Bogoliubov-de Gennes equations as before: ${ }^{10}$

$$
\sum_{j}\left(\begin{array}{cc}
\mathcal{H}_{i j, \sigma} & \Delta_{i j} \\
\Delta_{i j}^{*} & -\mathcal{H}_{i j, \bar{\sigma}}^{*}
\end{array}\right)\left(\begin{array}{c}
u_{j \sigma}^{n} \\
v_{j \bar{\sigma}}^{n}
\end{array}\right)=E_{n}\left(\begin{array}{c}
u_{j \sigma}^{n} \\
v_{j \bar{\sigma}}^{n}
\end{array}\right),
$$

except that here $H_{i j, \sigma}=-t-(\mu+\sigma h) \delta_{i j}+U_{0} \delta_{i, j_{0}}$ contains an impurity of strength $U_{0}$ located at $j_{0} . u_{j \sigma}^{n}$ and $v_{j \bar{\sigma}}^{n}$ are the Bogoliubov quasiparticle amplitudes on the $j$ th site. The selfconsistency condition for the OP,

$$
\Delta_{i j}=\delta_{j, i+\gamma} \frac{V}{4} \sum_{n} \tanh \frac{E_{n}}{2 k_{B} T}\left(u_{i \uparrow}^{n} v_{j \downarrow}^{n^{*}}+u_{j \downarrow}^{n} v_{i \uparrow}^{n^{*}}\right),
$$

is solved by iteration. Here $\gamma=( \pm 1,0)$ and $(0, \pm 1)$, and $\Delta_{i}$ $=\left(\Delta_{i+\hat{x}}+\Delta_{i-\hat{x}}-\Delta_{i+\hat{y}}-\Delta_{i-\hat{y}}\right) / 4$ is the $d$-SC OP at site $i$.

Previously we have studied the FFLO state in a clean system. ${ }^{10}$ We use one of the solutions as the initial configu- 
ration (but with all $\Delta_{j_{0}, j_{0}+\gamma}$ set to 0 ). Both strong unitary (i.e., $\left.U_{0}=100\right)$ and weak $\left(U_{0}=1\right)$ impurities have been investigated. We set $V=1.0, \mu=-0.4$, and $h=0.15$. The OP in a clean system has been plotted as Fig. 1(b) in Ref. 10.

In the presence of an impurity the OP still iterates practically to the same two-dimensional (2D) lattice in a $d$-SC, except for a local depression at the impurity site. The size of the order parameter hole created is of the order of the coherence length. Depending on the initial configurations, the iteration leads either to an OP saddle point being pinned at the impurity site, or, with slightly higher energy, an OP extremum, located at the center of a basic OP lattice bounded by four nodal lines, being pinned at the impurity site. ${ }^{14}$ When at an OP saddle point, the OP essentially vanishes at more sites around the impurity than when there is no impurity. When at an OP extremum, the OP vanishes on that site and is suppressed at its surrounding sites. (At the impurity site the OP practically vanishes for $U_{0}=100$, and is only suppressed by about $30 \%$ for $U_{0}=1$.)

Next, we calculate the LDOS near the impurity. The LDOS of spin-up and spin-down quasiparticles is given by

$$
\rho_{i \sigma}(E)=\sum_{n}\left[\left|u_{i \sigma}^{n}\right|^{2} \delta\left(E_{n}-E\right)+\left|v_{i \bar{\sigma}}^{n}\right|^{2} \delta\left(E_{n}+E\right)\right] .
$$

In what follows, we present the results for only spin-up quasiparticles since the spin-down LDOS spectra are simply the spin-up ones shifted to the right by $2 h$. The sum of spin-up and spin-down LDOS gives the total local differential tunnel conductance, measured by scanning tunneling microscopy with an unpolarized tip.

When an OP saddle point is pinned at the impurity site, Fig. 1(a) shows the spin-up LDOS on a nearest-neighbor site of the impurity, revealing the same four subgap peaks [marked by arrows, and confirmed by the maps in Figs. 1(b) and 1(c)], as discussed in Ref. 10, as well as the coherence peaks and a van Hove peak, but no new peak(s) that can be identified as the impurity-induced resonant peak(s). ${ }^{1}$ Comparing this figure with the LDOS plot at an OP saddle point in Fig. 4(b) of Ref. 10, we see that the outer two subgap peaks are perturbed only very weakly by the presence of a unitary impurity. (High-energy oscillations are caused by quasiparticles trapped between the impurities in the neighboring supercells. They weaken as $U_{0}$ is reduced.)

At first sight these results appear puzzling: According to Ref. 10, the inner two weak subgap peaks are due to the tails of the wave functions of ZEABSs, or midgap states, ${ }^{7}$ localized in this case near the halfway points between the center OP saddle point and its neighboring OP saddle points. Thus their weak dependence on the impurity potential near the center saddle point is not surprising. But the outer two strong subgap peaks can be identified as due to the finite-energy ABSs localized at the center saddle point. ${ }^{10}$ Why do these peaks also depend very weakly on the impurity potential right at this saddle point? Also, why are there no new resonant peaks induced by this impurity? The answer to these questions lies in the difference between the ABSs due to an OP well and the usual bound states by an ordinary potential well. For the latter, adding a strong impurity potential at the center of the potential well will certainly shift the energy of

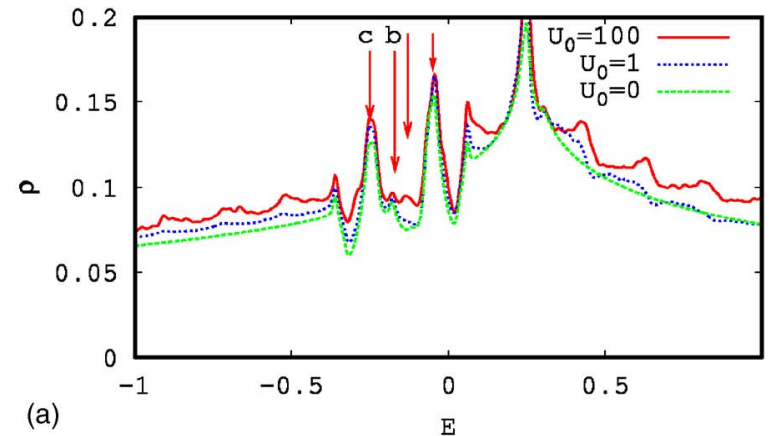

(b)
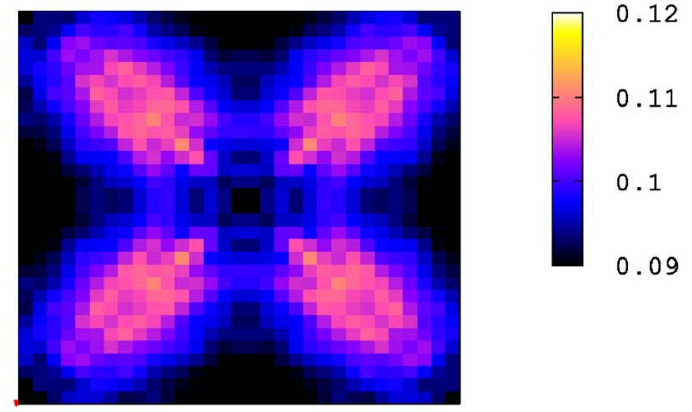

(c)

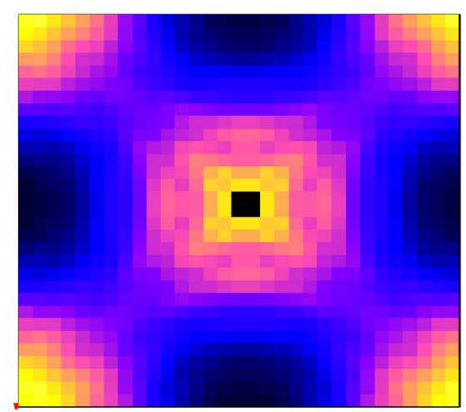

0.12

0.1

0.08

0.06

FIG. 1. (Color online) (a) The spin-up LDOS spectrum on a nearest-neighbor site of the impurity, which is at an OP saddle point. The subgap peaks of the LDOS are marked by arrows. (b), (c) The LDOS maps at the peak energies $E=-0.17$ and -0.25 , respectively, for $U_{0}=100$. In both maps, the impurity is at the center. The four corners of these maps are the neighboring saddle points of the OP.

the bound state, but for the former the quasiparticle is essentially moving at the Fermi momentum, and can be described by a semiclassical orbit. Without the impurity the orbit is a straight line segment shooting through the OP saddle point, with both ends terminated by Andreev reflections involving the same sign of the OP. The actual bound state is a coherent superposition of all such classical orbits of different orientations. With a unitary impurity at the saddle point, the classical orbit is deflected by the impurity to a new arbitrary direction (in coherent superposition) but the new OP value encountered has either two plus signs or two minus signs (one in momentum space since the OP is $d$ wave, and one in real space due to the FFLO state), leading always to no net sign change of the pair potential. After coherent superposition of all initial directions of the classical orbit, one can see that the resultant semiclassical bound-state wave function must be practically unchanged. (For a weaker impurity potential, the deflection probability is reduced, but the conclu- 


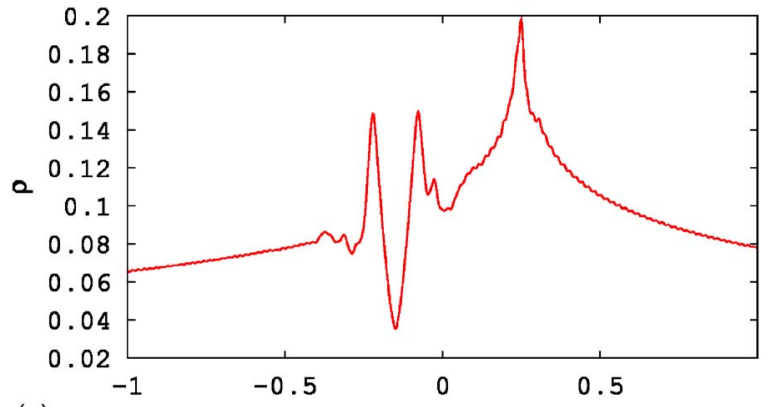

(a)
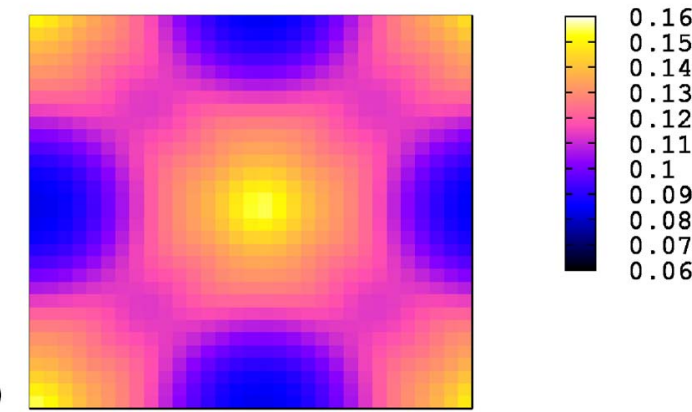

(b)

FIG. 2. (Color online) Similar to Fig. 1 except that the realspace sign change of the OP has been removed, and there is no impurity. In (a), the LDOS is calculated at a saddle point. Only two subgap peaks now appear near the two outer subgap peaks obtained in Ref. 10. (b) The LDOS map at one of the subgap peaks $(E=-0.22)$.

sion about the wave function remains practically the same.) This explains the weak dependence of the outer two subgap peaks on $U_{0}$, and why there are no impurity-induced NZERSs in this case, which requires seeing a sign change of the OP.

In order to test this reasoning, we change the OP to its absolute value (i.e., removing the real-space sign change but keeping the characteristics of the $d$-SC), and recalculated the LDOS at sites next to the original OP saddle point and its map, as shown in Fig. 2. Here only the two outer subgap peaks found in Fig. 1(a) appear, as confirmed by the map in Fig. 2(b) which shows the maximum intensity at a saddle point, but with slightly shifted energies, showing that these quasiparticle states do not result from the sign change of the OP in real space. Adding a unitary impurity to the center saddle point (not shown) actually makes the impurityinduced near-zero-energy resonant peak reappear, because the semiclassical scattering orbit can now encounter both signs of the OP.

Next we consider when an impurity is located at an OP extremum. In this case we might naively think that a unitary impurity can induce NZERSs in the LDOS just as in a uniform $d$-SC, ${ }^{1}$ since the impurity is in a local environment where the OP has a single sign in the real space, and changes sign only in the (relative) momentum space. However, Fig. 3(a) shows that two resonant peaks are induced by the unitary impurity at the LDOS minima or energy quasigaps of the host, and they are a pair separated by $\pm \epsilon_{0}$ before the Zeeman shift, with $\epsilon_{0}$ about $68 \%$ of the maximum gap for the parameter values considered. A strong resonance peak due to
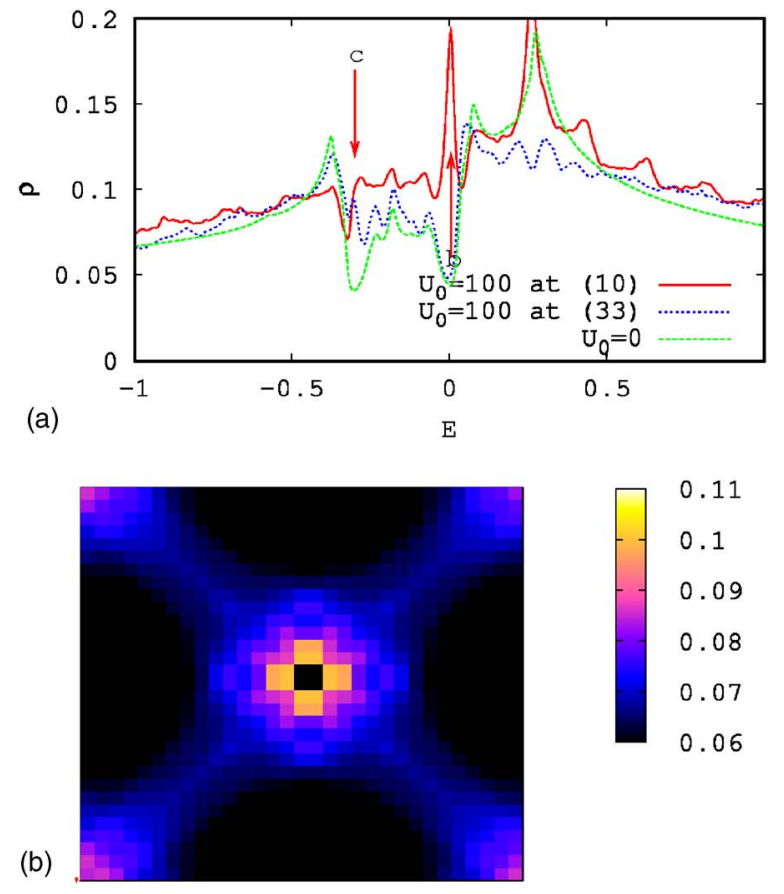

(b)

(c)

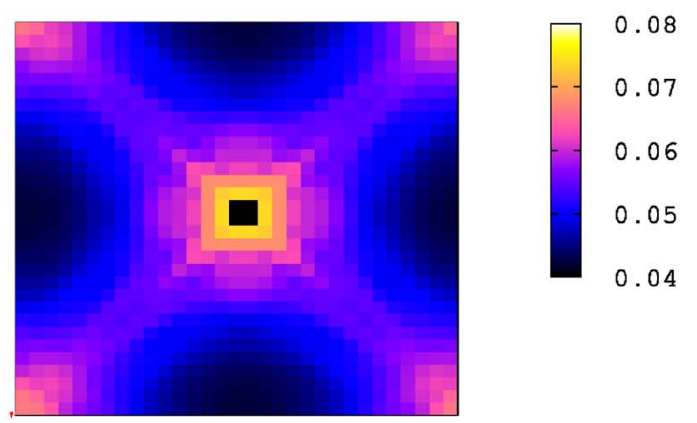

FIG. 3. (Color online) (a) The spin-up LDOS spectrum at the (10) and (33) sites relative to a unitary impurity at the (00) site, which is here also an OP extremum, revealing two impurityinduced resonant peaks, indicated by arrows marked for maps in (b) and (c), which confirm this interpretation. The $U_{0}=0$ curve is for a pure system. (b), (c) The LDOS map at peak energies $E=0.005$ and -0.305 , respectively, for $U_{0}=100$. In these maps, the impurity is located at the center. The four corners are maximum $|\Delta|$ sites in the neighboring cells of the 2D FFLO state.

the impurity located on the right of the subgap states of the pure FFLO state could be clearly seen at the (11) site relative the impurity at the $(00)$ site. The left resonant peak induced by the impurity is better revealed at the (33) site, where the other subgap peaks are lower. These resonant states are essentially localized around the impurity as is shown by the spatial maps calculated at these peak biases shown in Figs. 3(b) and 3(c). For a weaker impurity these impurity-induced peaks are even closer to the coherence peaks. These peaks are clearly located outside the subgap peaks of a pure FFLO state. ${ }^{10}$ Those subgap peaks are low in this plot as expected, since they are strong near the OP saddle points and halfway points between the neighboring saddle points only.

Separate calculations (with details omitted here) show that the unitary-impurity-induced resonant peaks are also at finite energies for $\Delta(\mathbf{r})=\Delta_{0} \cos (\mathbf{q} \cdot \mathbf{r})$, but are very near zero 
energy for $\Delta(\mathbf{r})=\Delta_{0} \exp (i \mathbf{q} \cdot \mathbf{r}) .{ }^{15}$ We offer the explanation below. The impurity-induced states are resonant states because their energies are outside the gap on the part of the Fermi surface near the nodal directions in the momentum space. But relative to the rest of the Fermi surface they are simply bound states localized near the vicinity of the impurity. It is well known that bound states can only form in the forbidden gaps of the continuum states. Now for the FFLO states, whether $1 \mathrm{D}$ or $2 \mathrm{D}$, there is already a band of continuum states centered at the gap center. ${ }^{10}$ This band is narrower for field closer to the lower critical field of the FFLO state, allowing the impurity-induced resonant peaks to also move toward the gap center. Also, this band is clearly absent in the current-carrying FF state, allowing the impurityinduced resonant peak to still appear as a NZBRP before the Zeeman shift.

Figures 3(b) and 3(c) also reveal that the impurityinduced resonant states have wave functions extending very far along the nodal directions of the $d$-wave OP. This extension can lead to couplings between the impurity-induced states localized in the neighboring supercells. But such couplings can only broaden the resonant peaks into narrow bands, and cannot cause repulsion between the two impurityinduced peaks.

In summary, we have studied the subtle effects of adding a very low concentration of unitary nonmagnetic impurities on the LTDOS in the vicinity of an impurity, if the system is in the FFLO state in a $d$-SC. A impurity in such a situation is locally stable at either an OP saddle point or an OP extremum. If the impurity is at an OP saddle point, then the LTDOS is practically unaffected by the impurity, with no impurity-induced resonant peak or peaks appearing, unlike in a uniform $d$-SC. If the impurity is at an OP extremum, then a $\pm \epsilon_{0}+$ (Zeeman energy) pair of finite-energy resonant peaks are induced in the LTDOS by the impurity, instead of one at near-zero energy, as in a uniform $d$-SC. The physics underlying these results has been expounded. Confirming these results can provide one of the strongest evidences for the existence of the FFLO state in a $d$-SC.

This work was supported by Grant No. E-1146 from the Robert A. Welch Foundation and by the Texas Center for Superconductivity at the University of Houston through the State of Texas.
${ }^{1}$ A. V. Balatsky, I. Vekhter, and Jian-Xin Zhu, Rev. Mod. Phys. 78, 373 (2006).

${ }^{2}$ A. V. Balatsky, M. I. Salkola, and A. Rosengren, Phys. Rev. B 51, 15547 (1995); A. V. Balatsky and M. I. Salkola, Phys. Rev. Lett. 76, 2386 (1996).

${ }^{3}$ S. H. Pan et al., Nature (London) 403, 746 (2000).

${ }^{4}$ J.-X. Zhu, T. K. Lee, C. S. Ting, and C.-R. Hu, Phys. Rev. B 61, 8667 (2000).

${ }^{5}$ J.-X. Zhu, C. S. Ting, and C.-R. Hu, Phys. Rev. B 62, 6027 (2000)

${ }^{6}$ I. Martin, A. V. Balatsky, and J. Zaanen, Phys. Rev. Lett. 88, 097003 (2002).

${ }^{7}$ C.-R. Hu, Phys. Rev. Lett. 72, 1526 (1994).

${ }^{8}$ I. Adagideli, P. M. Goldbart, A. Shnirman, and A. Yazdani, Phys. Rev. Lett. 83, 5571 (1999); S. Ryu and Y. Hatsugai, ibid. 89, 077002 (2002).

${ }^{9}$ P. Fulde and R. A. Ferrell, Phys. Rev. 135, A550 (1964); A. I. Larkin and Yu. N. Ovchinnikov, Sov. Phys. JETP 20, 762 (1965). See also, for example, L. G. Aslamzov, ibid. 28, 773 (1969); H. Shimahara, J. Phys. Soc. Jpn. 67, 736 (1998); D. F. Agterberg and K. Yang, J. Phys.: Condens. Matter 13, 9259 (2001); K. Maki and H. Won, Physica B 322, 315 (2002); A. B. Vorontsov, J. A. Sauls, and M. J. Graf, Phys. Rev. B 72, 184501 (2005), etc.

${ }^{10}$ Q. Wang, H.-Y. Chen, C.-R. Hu, and C. S. Ting, Phys. Rev. Lett. 96, 117006 (2006).

${ }^{11}$ A third type can potentially appear at surfaces and interfaces, which is not considered in this study.
${ }^{12}$ A. Bianchi, R. Movshovich, C. Capan, P. G. Pagliuso, and J. L. Sarrao Phys. Rev. Lett. 91, 187004 (2003); H. Radovan et al., Nature (London) 425, 51 (2003); C. Capan, A. Bianchi, R. Movshuvich, A. D. Christianson, A. Malinowski, M. E. Hindley, A. Lacerda, P. G. pagliuso, and J. L. Sarraw, Phys. Rev. B 70, 134513 (2004); T. Watanabe, Y. Kasahara, K. Izawa, T. Sakakibara, Y. Matsuda, C. J. Van der Beek, T. Hanagwri, H. Shishido, R. Settoi, and Y. Onuki ibid. 70, 020506(R) (2004); C. Martin, C. Mavtin C. C. Agosta, S. W. Tozer, H. A. Radovan, E. C. Paln, T. P. Murphy, and J. L. Sarrao, ibid. 71, 020503(R) (2005); K. Kakuyanagi, M. Saitoh, K. Kumagai, S. Takashima, M. Nohara, H. Takagi, and Y. Matsuda, Phys. Rev. Lett. 94, 047602 (2005).

${ }^{13}$ K. Izawa, H. Yomaguchi, Yuji Matsuda, H. Shishi do, R. Settai, and Y. Onuki Phys. Rev. Lett. 87, 057002 (2001); C. Petrovic et al., J. Phys.: Condens. Matter 13, L337 (2001); R. Movshovich, M. Jaime, J. D. Thompson, C. Retrovis, Z. Fisk, P. G. Pagliuso, and J. L. Serraw, Phys. Rev. Lett. 86, 5152 (2001); C. Petrovic, S. L. Budko, V. G. Kogan, and P. C. Canfield, Phys. Rev. B 66, 054534 (2002); P. M. C. Rourke, M. A. Tanatar, C. S. Turel, J. Berdeklis, C. Petrovic, and J. Y. T. wei, Phys. Rev. Lett. 94, 107005 (2005).

${ }^{14}$ The percentage difference in excess energy due to the impurity is about $1 \%$ for $U_{0}=100$, and about $6 \%$ for $U_{0}=5$.

${ }^{15}$ J.-X. Zhu, C. S. Ting, and C. W. Chu, Phys. Rev. B 62, 11369 (2000). 